\title{
Living in interesting times - challenging protein metabolism in the era of the epidemiological shift
}

\author{
Alessandro Laviano
}

The recently released results of the Global Burden of Disease, Injuries, and Risk Factor study 2013 reveal that during the past 23 years, metabolic and behavioral risk factors, including high BMI, increased their impact on global mortality and disability-adjusted life-years [1]. These data highlight the importance of the investigation of the metabolic impact of specific nutrients and different eating behaviors on preserving and restoring the health of individuals and also populations.

Protein metabolism is a key component of human metabolism and is closely related to health and resistance to exogenous and endogenous insults. Acute and chronic diseases are characterized by the progressive derangement of body composition, which is secondary, among other factors, to increased proteolysis without the compensatory increase of muscle anabolism [2]. The resulting muscle loss is therefore the phenotype of the molecular changes induced by the presence of a disease. However, the molecular changes of protein metabolism also result in clinically relevant conditions. Muscle mass has been consistently demonstrated to predict the rate of complications during acute and chronic diseases. In particular, low muscle mass, that is, sarcopenia, is associated to treatmentrelated toxicity in cancer patients receiving chemotherapy or undergoing surgery [3,4]. Muscle wasting has been for long considered unresponsive to nutritional intervention because of inflammation-driven anabolic resistance [5]. Emerging and convincing data now suggest that muscle wasting can be effectively prevented and treated by delivery of adequate amount and specific types of amino acids (Engelen et al., pp. 39-47), which, in turn, may enhance the efficacy of anticancer treatments. Thus, anabolic resistance of diseased states may actually be overcome by timely and specific protein delivery.

On the contrary, disease-related early changes of protein metabolism can be exploited as markers of developing diseases. In this light, changes in plasma branched-chain amino acid levels are closely linked to the risk of developing diabetes (Giesbertz and Daniel, pp. 48-54), whereas alterations of tryptophan metabolism could be considered as markers of neuroinflammation (Strasser et al., pp. 55-61). The progressive elucidation of the early changes of protein metabolism and their relationship with the risk of developing chronic diseases will increase the efficacy of preventive and therapeutic strategies. Nevertheless, these clinical achievements will not be possible without a thorough assessment of the interrelationships between different amino acid metabolic pathways (Marini, pp. 62-66) and the precise detailing of the mechanisms of amino acid sensing (Ham et al., pp. 67-73).

Prevention of diseases is a key target for basic and clinical research in the field of metabolism. Whether specific dietary behaviors could yield to healthy aging remains to be demonstrated in large, prospective, randomized, clinical trials. However, data emerging from animal studies could be verified in large epidemiological databases, even if this methodological approach cannot replace prospective studies. Significant scientific interest has been devoted to protein intake as a dietary strategy to slow aging and maintain functional independence. However, recent analysis of epidemiological databases suggests that high protein intake reduces mortality in older adults, whereas it is associated to increased mortality in younger adults (Mirzaei et al., pp. 74-79). These results disclose the complexity of human metabolism and underline the need for carefully considering different factors before interfering with it by using sometimes extreme dietary strategies.

Life expectancy is gradually increasing, this being associated with the progressive increase of chronic and degenerative diseases, including cancer [6]. Favoring the healthy aging of human

Department of Clinical Medicine, Sapienza University, Rome, Italy

Correspondence to Alessandro Laviano, MD, Department of Clinical Medicine, Sapienza University, viale del Policlinico 155, 00161 Rome, Italy. Tel: +39 0649973902; fax: +39 064440806;

e-mail: alessandro.laviano@uniroma1.it

Curr Opin Clin Nutr Metab Care 2016, 19:37-38

DOI:10.1097/MCO.0000000000000244 
population remains the imperative goal to not only preserve the economic sustainability of healthcare systems but also maintain social stability. The better understanding of human metabolism and, in particular, its interactions with the external, that is, nutrients, pollutants, and others, and the internal environment, that is, microbiota, oxidative stress, and others, will pave the way to new and effective preventive and therapeutic strategies. In this light, exciting data are emerging from the study of protein metabolism at different ages and after different challenges. And more is to come.

\section{Acknowledgements}

None.

\section{Financial support and sponsorship}

None.

\section{Conflicts of interest}

There are no conflicts of interest.

\section{REFERENCES}

1. Forounzafar MH, Alexander L, Anderson HR, et al. GBD 2013 Risk Factors Collaborators. Global, regional, and national comparative risk assessment of 79 behavioural, environmental and occupational, and metabolic risks or clusters of risks in 188 countries, 1990-2013: a systematic analysis for the Global Burden of Disease Study 2013. Lancet 2015. [Epub ahead of print]

2. van Dijk DP, van de Poll MC, Moses AG, et al. Effects of oral meal feeding on whole body protein breakdown and protein synthesis in cachectic pancreatic cancer patients. J Cachexia Sarcopenia Muscle 2015; 6:212-221.

3. Mir O, Coriat M, Blanchet $\mathrm{B}$, et al. Sarcopenia predicts early dose-limiting toxicities and pharmacokinetics of sorafenib in patients with hepatocellular carcinoma. PLoS One 2012; 7:e37563.

4. Lieffers JR, Bathe OF, Fassbender $\mathrm{K}$, et al. Sarcopenia is associated with postoperative infection and delayed recovery from colorectal cancer resection surgery. Br J Cancer 2012; 107:931-936.

5. Fearon K, Strasser F, Anker SD, et al. Definition and classification of cancer cachexia: an international consensus. Lancet Oncol 2011; 12: 489-495.

6. Ahmad AS, Ormiston-Smith N, Sasieni PD. Trends in the lifetime risk of developing cancer in Great Britain: comparison of risk for those born from 1930 to 1960 . Br J Cancer 2015; 112:943-947. 